\title{
Optimizing the Technological Design of a Blended Synchronous Learning Environment
}

\author{
Lauren Angelone \\ Xavier University \\ Zachary Warner and Janet Mannheimer Zydney \\ University of Cincinnati
}

\begin{abstract}
Blended synchronous learning has the potential to increase students' co-presence and improve upon the flexibility and accessibility of course offerings if designed well. This method of instruction, however, has yet to realize that potential. This exploratory study used qualitative methods to iteratively design, assess, and refine the technological design of a blended synchronous learning environment to improve the learner experience. Across three iterations, a combination of qualitative data collection and analysis procedures were used to examine the influence of design decisions on the experiences of the students and the instructor. The findings resulted in a set of design recommendations that can serve as a guide for future research on the technological design of blended synchronous learning environments.
\end{abstract}

Keywords: blended synchronous learning, web conferencing, qualitative case study, copresence

Angelone, L., Warner, Z., \& Zydney, J.M. (2020). Optimizing the technological design of a blended synchronous learning environment. Online Learning, 24(3), 222-240. https://doi.org/10.24059/olj.v24i3.2180

\section{Optimizing the Technological Design of a Blended Synchronous Learning Environment}

Blended synchronous learning, also referred to as hybrid synchronous instruction (Romero-Hall \& Vicentini, 2017), HyFlex course design (Para \& Abdelmalak, 2016), synchronous hybrid learning (Butz \& Stupnisky, 2016), and synchronous online teaching (Park \& Bonk, 2007) integrates online and face-to-face instruction to create learning environments where students can attend in-person or from a distance simultaneously. This type of learning environment has been used more frequently in higher education to accommodate student desires for both flexibility and personal connection (Bower, Delgarno, Kennedy, Lee, \& Kenney, 2015), and now that universities are trying to accommodate for socially distanced classrooms the use of this environment is surging. For decades, distance learning provided the necessary flexibility for students to pursue higher 
education in a variety of ways (Moore, 2013). However, students learning online often miss the personal connections made in the classroom. Blended synchronous learning is a potential solution to this problem, but questions remain about how to best leverage technology in a way that creates a seamless experience where learners near and far can connect.

The term co-presence is used to describe the feeling of being together in virtual environments (Bulu, 2012). In order to create a seamless blended synchronous experience where co-presence can thrive, the technological design must be carefully considered as technological issues are a common problem in this type of environment (Cunningham, 2014; White, Ramirez, Smith, \& Plonowski, 2010; Wang, Huang, \& Quek, 2018; Bower et al., 2015). The evolution of web conferencing tools designed to allow for online meetings between individuals in separate locations may support co-presence in blended synchronous learning environments by mitigating the technological issues experienced in the past. This study sought to explore the optimal technological design of a blended synchronous learning environment leveraging a webconferencing platform by examining the impact of design decisions on co-presence across multiple iterations.

\section{Background}

The technological design is crucial for setting the foundation for learning and social interaction within blended synchronous learning (Wang \& Huang, 2018). Two goals of the technological design are to create a seamless learning experience and a sense of co-presence among learners, which for the purposes of this study includes the instructor.

\section{Seamless Learning Experiences in Blended Synchronous Learning}

One focus of the technological design in blended synchronous environments has been to determine how to create a seamless learning experience to connect on-campus and online learners (Bower et al., 2015). "Seamless learning refers to the seamless integration of the learning experiences across various dimensions" (Wang \& Looi, 2011). Although more often discussed in the context of mobile learning, the construct of seamless learning aligns well within the context of blended synchronous learning given the varying dimensions created by different participant locations and a range of communication modes utilized. Unfortunately, creating a seamless learning experience is often unattainable because of frequent technology issues or steep learning curves associated with using the technology (Bower et al., 2015). Frequently reported technology issues when using these tools include unreliable connections (Cunningham, 2014), lag times (White et al., 2010; Wang et al., 2018), and audio issues (Bell, Sawaya, \& Cain, 2014; Bower et al., 2015; Para \& Abdelmalak, 2016; Park \& Bonk, 2007).

The technological design can be improved by taking into consideration both design and implementation factors as recommended by Bower et al. (2015) in their blended synchronous learning design framework. For example, before the session, these researchers recommend testing the technology in advance (Bower et al., 2015). In addition to taking steps to reduce technical issues, Wang and Huang (2018) recommend that the tools and equipment be selected to provide clear visual and audio connections. Recommended strategies include using mobile devices for presentation and multiple microphones that are muted when not in use (Wang and Huang, 2018). In addition, during implementation, Bower et al. (2015) note that training participants on how to use and troubleshoot the technology has been found to help create a more seamless learning experience. Seeking technology assistance through using co-instructors (Bell et al., 2014) or teaching assistants (Cunningham, 2017; White et al., 2014) can also be helpful. 


\section{Co-Presence in Blended Synchronous Learning}

Another goal for blended synchronous learning is to establish co-presence among learners. Designers have experimented with a variety of equipment, tools, and class groupings to create this feeling. Some equipment that helps to create co-presence are multi-screen projections (Szeto, 2015; Szeto \& Cheng, 2016); multiple cameras that provide different views of the class (Bell et al., 2014; Wang, Quek, \& Hu, 2017); and devices, such as iPads or monitors, set up to display an individual (Bell et al., 2014; Cunningham, 2014).

Some tools have affordances that can also help with creating a sense of co-presence. For example, web conferencing tools that allow for multiple modalities, such as video, audio, and text, help students contribute to the discussion in a way that is most comfortable to them (Wang \& Huang, 2018). Web conferencing tools that have been reported in the literature on blended synchronous learning include Adobe Connect, Blackboard Collaborate, Centra, Google Hangouts, GoToMeeting, Skype, and Zoom. One web conferencing tool that has not been studied within the context of blended synchronous learning is WebEx. Based on testing by this study's university technology personnel, WebEx limited lag time issues compared to other tools. Thus, the current study examined the use of WebEx to support blended synchronous learning.

Finally, the class groupings themselves can sometimes help with co-presence, such as partnering on-campus and online students (Bell et al., 2014; Cunningham, 2014; Wang \& Huang, 2018; Wang et al., 2017; 2018) or using small groups with a mixture of students participating oncampus and online (Bell et al., 2014; Bower et al., 2015; Park \& Bonk, 2007).

Although the intention of designs leveraging these elements is to create a sense of copresence, the actual feelings of students are often mixed. For example, many designs can feel awkward to the on-campus students who need to do things they wouldn't ordinarily do in the classroom, such as move an iPad so the online student can see better (Bell et al., 2014; Cunningham, 2014) or speak directly into a microphone (Szeto \& Cheng, 2016; Wang, Quek \& $\mathrm{Hu}, 2017)$. In some instances, these extra tasks were met with reluctance or even resentment among on-campus students (Cunningham, 2014) or made the online students feel dependent or unwanted (Bell et al., 2014; Cunningham, 2014). Some designs end up improving the presence of one group at the sacrifice of the other. For example, having each online student displayed on an individual device helped the on-campus students feel more connected to the online students, but resulted in the online students feeling more disconnected from one another (Bell et al., 2014). Some studies

have noted that students tend to feel less connected with students attending in the opposite mode (Bower, Lee \& Delgarno, 2017; Butz \& Stupnisky, 2016).

Given these mixed results, more work is needed experimenting with the technological design to help achieve the goals of creating a seamless learning experience and a sense of copresence. Since technology has proven to be an issue that impacts the intended goals of blended synchronous learning environments, this study focuses on how to improve the technological design to facilitate better experiences for learners. 


\section{Methods}

\section{Case Study}

The current study uses a case study approach as described by Yin (2003). The unit of analysis for this case is a graduate level course designated as here or there (HoT) in which the course was mostly online with four blended synchronous sessions that students could attend in person or virtually via WebEx (Zydney, McKinney, Lindberg \& Schmidt, 2019). The instructional format of the blended synchronous session was similar for all sessions. Each session began with a whole class activity or lecture, then the students met in breakout groups to have discussions about the course material, and the class ended with a whole-class debrief with one student from each group sharing what had been discussed in the small groups. The technology for each session, however, was iterated upon after collecting feedback from students. The breakout groups were facilitated by a student facilitator who used a protocol to guide the flow and timing of the discussion in order to create equity of participation, encourage different perspectives, and prompt reflection (McDonald, Zydney, Dichter \& McDonald, 2012). For additional details on the pedagogical approach and how it was enhanced during the sessions, please see Zydney, Warner, and Angelone (2020).

This study focuses on the technological design of a blended synchronous environment, using an iterative approach to better understand how the design influenced the instructor and learner experience. A case study is appropriate for this study as the research question is both exploratory and contextual in nature. The research question for the current study is:

- How does the technological design of a blended synchronous learning environment influence the learner experience?

\section{Participants}

The graduate level course under study included 16 participants that consented to be a part of the study from a large midwestern university (12 females and 4 males). All 17 students in the course were informed about the research by the instructor and then a research assistant followed up to obtain consent. The participants included K-12 teachers, higher education administrators and faculty, a corporate manager, and a doctoral student. The instructor was both the principal investigator and a participant in the research, consistent with more participatory methods in which researchers are included in the research rather than presupposing that an objective distance can eliminate bias (Tolman \& Brydon-Miller, 2001). No race data was collected. This project was approved by the Institutional Review Board.

The HoT format allowed participants to choose to come to campus (here) or participate virtually (there). Across the sessions, approximately half of the students chose one mode of participation and continued to use the same mode throughout. Three students participated in both modes. No students had taken a blended synchronous course before. Five students rated themselves as novice users of WebEx and two rated themselves as experts, with the remainder falling somewhere in between.

\section{Data Collection and Analysis}

Multiple data sources were utilized to enhance data credibility (Yin, 2003). Data collected included field notes of observations from each blended synchronous session, debrief sessions, survey responses, interviews with participants, and a member check. Field notes were collected 
during each blended synchronous session using a template (Appendix A) based on the work of Merriam (2001). Surveys were designed by the research team to understand learner experience in the HoT sessions. Survey questions included items regarding how the students participated in the session, what supported their communication, what challenges they experienced, and suggestions to improve the HoT sessions. These surveys were used to make technological design changes over each iteration and then studied as part of the data corpus to understand instructor and learner experience. Surveys were sent to all students after each iteration by the research assistant. Thirteen students responded in the first iteration, ten in the second iteration, and four in the third iteration. The decreased participation was likely due to the voluntary nature of participating in the survey but could also be attributed to the decrease in issues experienced by the students over time. Interviews were conducted using a script (Appendix B) based on the simulated recall method (Dempsey, 2010). An instructor interview was conducted midway through the course and four student interviews (one online and three on-campus) were conducted by the research assistant after the third blended synchronous session. The instructor was included in the research as her perceptions and reflections gave insight into technological design changes that could impact learner experience. Finally, a member check was conducted by a student who had attended the blended synchronous sessions both online and on-campus.

The data was then transcribed as necessary and analyzed using an interpretive methodology as described by Erickson (1986). The research team, which included the instructor, their graduate assistant, and a consultant, reviewed the data and generated assertions using a quasi-grounded theoretical approach (Charmaz, 2006). The research team came together multiple times to validate or disprove assertions before the final analysis of the entire data corpus took place.

The following section includes a description of each iteration of the blended synchronous sessions and details the data collected and technological design decisions made as a result of the instructor and learner experience.

\section{In-the-Moment Design Decisions}

\section{Results}

First Iteration Initial Design. The initial technological design leveraged recommendations from the blended synchronous literature for creating co-presence and included design decisions regarding the equipment, tools, and classroom configuration. The initial technological design along with subsequent iterations of the design are displayed in Table 1 below.

In the first iteration, all students (both online and on-campus) logged into a WebEx training session that enabled everyone to see one another when the whole class was together. To capture the video in the room, a swivel camera connected wirelessly to a pendant worn by the instructor directed where the camera pointed. Multiscreen projection was provided through two projectors, a SMART Board, and six TV monitors at the end of each group table. One projector displayed the online participants, one projector displayed the chat, and the SMART Board/TV monitors displayed a PowerPoint presentation. Within the classroom, one central speaker microphone was set up on a rolling chair so that it could be moved closer to the on-campus students to allow online students to hear. For small group discussions, the instructor created breakout groups within the WebEx training session that she controlled. Both on-campus and online students used headsets to participate in the small group discussions and turned their microphone on when they wanted to speak. 
Table 1

Technological Design Changes Across the Iterations

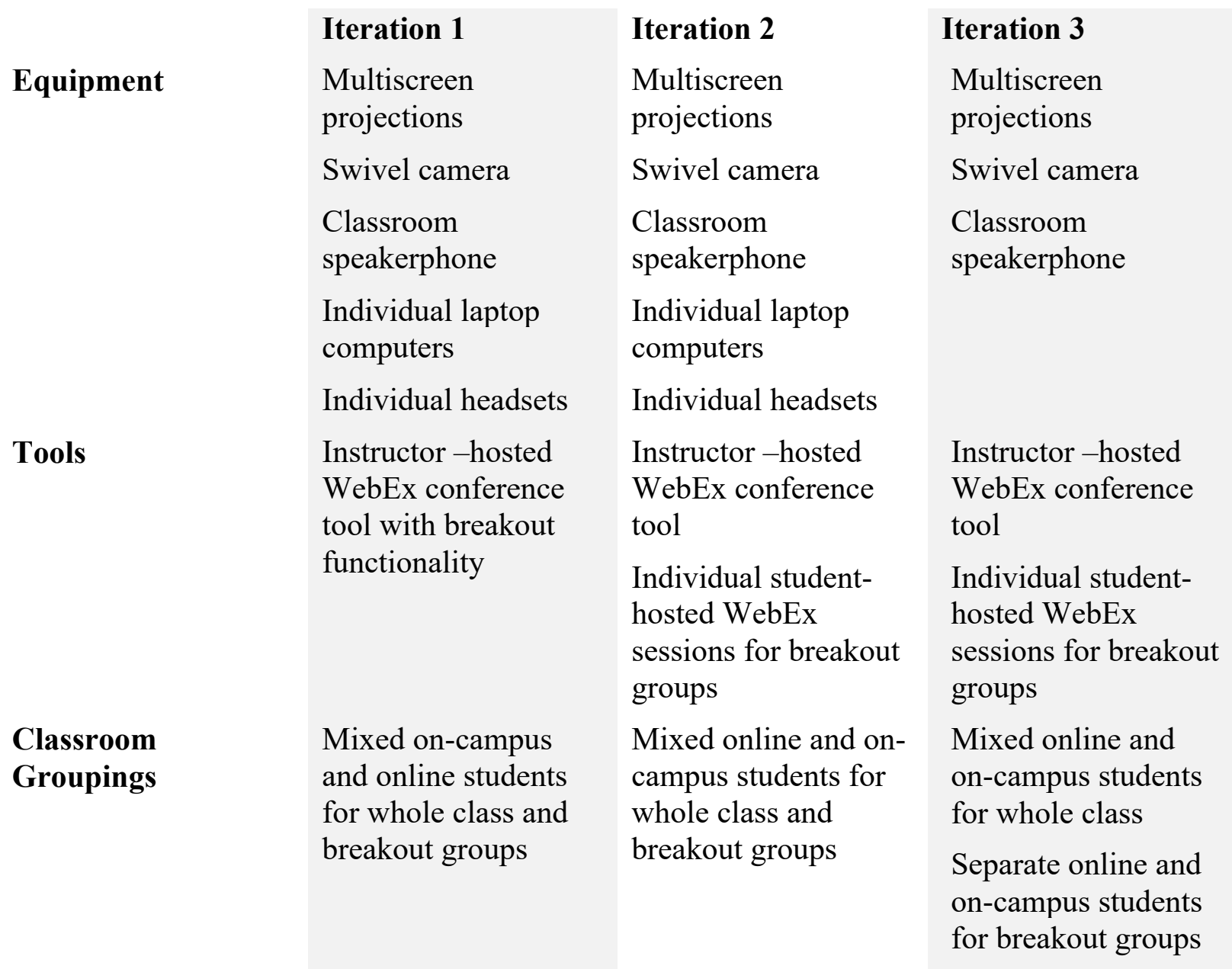

First Iteration Feedback. Thirteen students responded to a survey requesting feedback after the first iteration. Positive feedback included seeing the discussion prompts on the TV monitors in the classroom, using the chat functionality in WebEx, and having on-site technology support. Most of the issues noted involved audio: 11 of the 13 students mentioned audio as a problem, frequently citing background noise and audio feedback. Other technical issues included several problems with the WebEx breakout functionality, such as the inability for students to use their webcams during small group discussions.

There were also several timing and communication issues noted. The troubleshooting process created downtime. One student commented, "The small group breakouts seemed to take a lot of time and required a lot of coordination to implement." The instructor had difficulty communicating to the whole group during the breakout session and either had to use the text messaging system, which limited the number of characters that could be sent or had to relay information through the student facilitators. An online student cited this as an obstacle, commenting "I had to wait until a person who was there relayed the information to me." The 
instructor also had difficulty monitoring the online chat while teaching and missed some messages from students who were having difficulty.

Second Iteration Design Changes. Based on feedback from the first session, several design changes were made. Given the issues with the WebEx breakout functionality, studenthosted WebEx meeting rooms were used for the breakout sessions. Everyone joined the main WebEx training session for the whole class introduction activity and the debrief, but then logged out of the main session and joined separate meeting rooms hosted by the student facilitators. This shift enabled students to use video during the breakout, allowing them to see one another more. The introductory activity also gave everyone an additional opportunity to be on camera. The oncampus students passed the pendant that directed where the swivel camera pointed, and online students passed a virtual ball within WebEx to present. The instructor encouraged online students to use their webcam, although some students opted not to. To resolve the audio issues related to background noise caused by having the speaker microphone on the rolling chair, on-campus students sat in a tight circle around the speaker microphone during the whole-class discussions. To improve communication, the instructor logged into each student-hosted session for a short period. Students were also given a phone number to call if they had technical issues so that they were not limited to the chat. Finally, an on-campus student was assigned the role of "chat monitor" to alert the instructor if there was a chat message that required her response.

Second Iteration Feedback. Ten students responded to a survey asking for feedback on the second iteration. Positive student comments included appreciation of fewer technology issues, use of student-hosted breakout sessions, and ability to see more online students.

Although many acknowledged that there were fewer technology issues as a result of the student-hosted sessions, some students were displeased, as one wrote "Having to sign out of the class WebEx session and into a completely new session for the breakout groups was a bit of a hassle. I would prefer just doing the breakout groups through the class WebEx." In addition, there were two students who did not successfully navigate to their respective breakout session and missed the remainder of the class as a result. The instructor commented in an interview after this session that she continued to feel overwhelmed by the technology and the need to troubleshoot. Despite the improvements made to timing and communications, one student reported feeling rushed because of technology problems, and two students missed the directions that provided the phone number to call for technical issues.

Observations indicated that the most ineffective aspect of the class was the introductory whole-class activity. Passing the pendant for on-campus students and the virtual presentation ball for online students caused delays, which made the discussion feel unnatural. Moreover, on-campus students had an additional inconvenience of having to stand up when sharing due to the angle of the swivel camera, which was designed to capture the movement of an instructor standing for a presentation, as opposed to students sitting in a circle.

Third Iteration Design Changes. To improve communication, instructions were emailed and posted in several places before and during class. In order to cut down on the technical issues and reduce the number of things the instructor needed to attend to simultaneously, students were divided into groups of either all on-campus students or all online students. These class groupings made it possible to reduce the technology in the classroom because on-campus students did not need their own devices or headsets. The introductory whole-class activity was designed to remove the need to pass the pendant or virtual ball. The swivel camera was set up on a tripod to allow on- 
campus students to remain seated while talking. During the debrief, on-campus students used a physical whiteboard to share their results whereas the online students shared their results orally.

Third Iteration Feedback. Because this was the last iteration of the study, students were asked to comment on their experience in the sessions as a whole. Four students responded to the final survey. Students commented that the biggest improvements over the course of the semester were fewer technology problems, better time management and flow, improved comfort with technology, and separating the students by attendance mode. Students felt that the persistent challenges over the semester were transitioning between different technologies, connectivity issues, and keeping online students engaged.

Observations indicated that there was less tension in the classroom during the third iteration. The instructor seemed more relaxed, and there were numerous instances of laughter and jokes, indicating everyone's increased comfort level. Minor technical issues were resolved quickly. The on-campus students appeared excited to use the physical whiteboards to share their ideas. Online students asked questions "seamlessly" during the introductory whole-class activity. After the session, the instructor and research assistant who observed the classroom debriefed the session and concluded that the session went as well as possible with the technology available.

\section{Technological Design Assertions}

Utilizing the data corpus, the design team iteratively reviewed the data and generated assertions (Erickson, 1986) based on common themes using a quasi-grounded theoretical approach (Charmaz, 2006). These assertions make the following claims about the data.

Technology as a Limitation. As described in the previous section, the instructor employed numerous tactics throughout the iterations to create an engaging learning environment for both oncampus and online students. Although the design decisions led to marked improvements in the learner experience after the first iteration, technology often acted as a limiting factor. For example, multiple microphones in the classroom caused audio interference and the swivel camera created unnatural delays in the discussion, both requiring additional class time to familiarize students with the equipment. Also, some students found WebEx to not be user friendly and had difficulties navigating between sessions. During her interview, Lucy ${ }^{1}$, an on-campus student, summarized the effect technology had on her experience in saying, "I was really surprised at how technology almost interrupted it. I was so looking forward to being in virtually with everybody and I felt like the technology just wasn't there for it." Despite these limitations, some of the challenges may have been due to the students' lack of familiarity with the tools. Lucy also stated in her interview, "just using WebEx was a big learning curve for me." Furthermore, students who had worked with webconferencing tools previously showed some frustration with their classmates' need to acclimate, as one student wrote after the first iteration, "The experience could have been much better if WebEx had been used correctly."

Awkwardness in Merging Environments. There is an awkwardness in merging oncampus and online learning environments. Whether attending a blended synchronous environment on-campus or online, all students are experiencing a mediated classroom that requires learning how to use the technology. The mediation, while expected by the online students, appeared to be more challenging for the on-campus students who anticipated a more typical classroom experience.

\footnotetext{
${ }^{1}$ Pseudonyms used for all student names
} 
On-campus students felt awkward using unnecessary technology to communicate with fellow on-campus students. In the first two iterations, online and on-campus students all wore headsets and interacted with one another through WebEx. This created an unnatural way for oncampus students seated together to communicate with one another. When asked about the challenges of the first session in a survey, one anonymous on-campus student stated that "many moving parts were sometimes difficult to navigate (when to use my headset vs. when to speak aloud)."

In trying to include online students in a more authentic way, on-campus students were often forced to mediate an experience that would not typically need any mediation. For example, passing the pendant to direct the swivel camera was seen as awkward by the on-campus students (see Figure 1). Mike, an on-campus student, remarked: "What I felt was the most ineffective part for lack of a better word... was standing in front of the class and passing the little ball around and presenting." For online students, the mediation of their experience seemed to be more expected. For example, George praised the breakout sessions during earlier iterations when on-campus students were initially frustrated. He noted: "I think you get better connections in the smaller breakout sessions than in the large group."

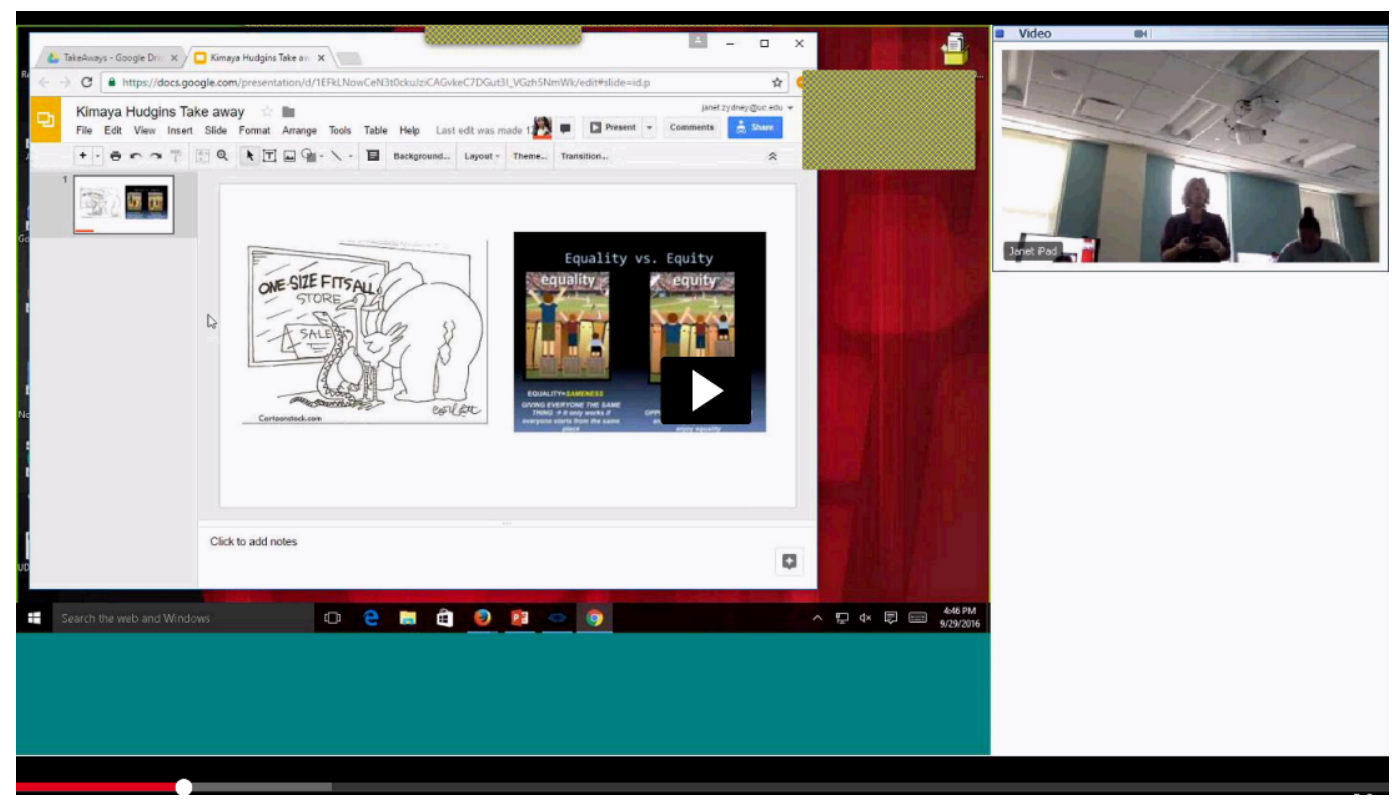

Figure 1. Screenshot from WebEx showing on-campus standing and holding a pendant to direct the swivel camera.

Experience with the technology also seemed to help eliminate some of the awkwardness of merging the environments. George, who was also a teacher in an online school, suggested that his background allowed him to more seamlessly lead and participate in sessions than those with no experience. In addition, his expectations of the blended synchronous environment were more tempered because he was familiar with the online technology. He explained, "Yeah, there's a lot going on. I mean, especially for someone if they're new. I mean, I volunteered because of the online environment and being comfortable with it."

Vulnerability of Transitions. Class momentum, and ultimately student engagement, was most vulnerable during transitions. Starting the sessions and switching between sessions was consistently an issue regardless of the approach used. 
Starting the sessions proved challenging for the learners in multiple ways. First, setting up the technology required the instructor, a research assistant, and a university technician to arrive at least an hour prior to each session. Setup was so time-consuming that it often carried over into the first several minutes of scheduled class time, leading one student to comment following the second iteration, "Starting the class still seems to be difficult [and] time consuming." However, preparing the technology was not just a challenge for the instructor, as students frequently required help logging into the WebEx meeting or troubleshooting audio or video issues with their laptops. One student commented, "I cannot get to the session 15 minutes before to get everything set up."

Students shared a similar sentiment when discussing transitioning to the breakout sessions. For example, one student stated there were "significant issues" starting the breakout sessions and another added "It took us a long time to get all of the people in the breakout room. I think we lost 10 minutes." Some evidence suggested the transitions affected the students' overall experiences. For example, Mike stated, "when you have a couple [of technology issues] like that at the beginning of the session it's hard to get engaged." He added that he recalled thinking during the sessions "I'm engaged now but am I going to have another technical issue?"

Seeing is Believing. Participants had a more authentic experience when they were either face-to-face or using video. The desire for the visual was strong enough to make the case that a better simulation of the physical classroom is needed to create a seamless experience in a blended synchronous context.

This desire for visual connections varied along with the design changes involving the use of WebEx and the physical classroom configuration. After the first iteration, several students discussed their desire to see students more. For example, one anonymous student mentioned that it was a challenge that "most did not use video" during the whole-class discussions (see Figure 2), and another cited the problem of "not being able to have video during breakouts." These issues were resolved in later iterations by encouraging more online students to use their webcams during whole-class discussions and using student-hosted meeting rooms that enabled video for the breakout discussions.

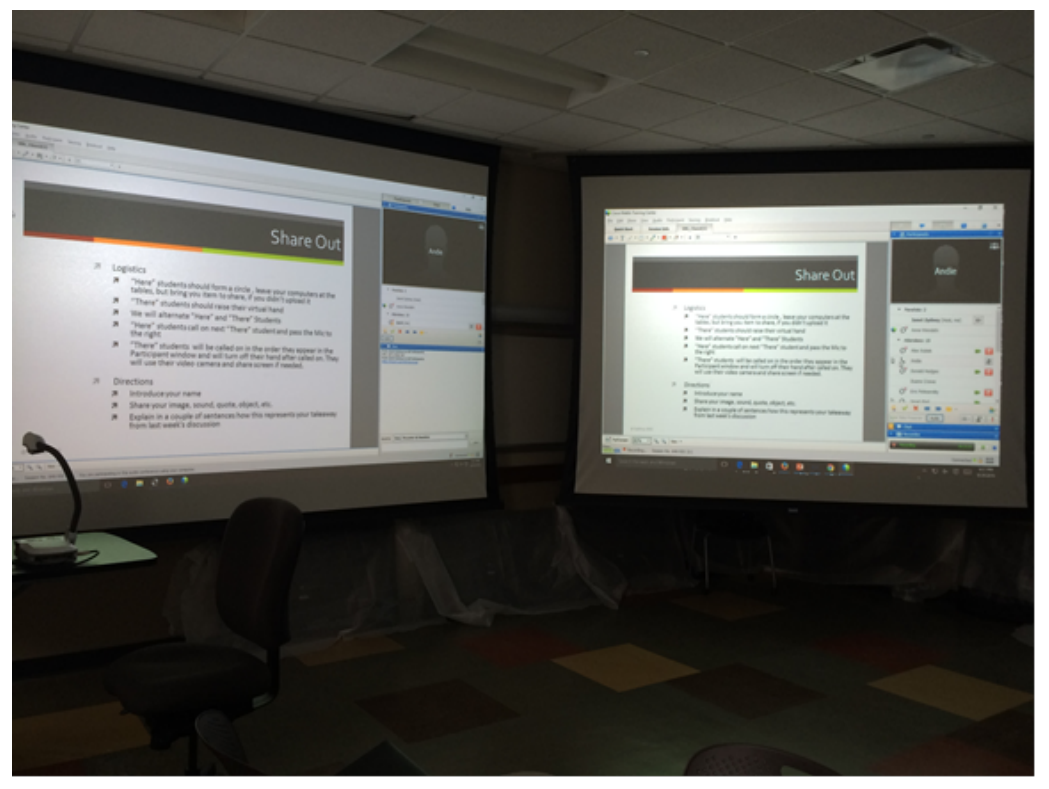

Figure 2. The projection screens in class displaying WebEx as an online student was presenting without video (top right of each screen). 
Although enabling video helped to some extent, it did not fully satisfy the need for students to see and interact with one another. George discussed that he felt less connected to others as an online student, in part due to the lack of informal bonding with classmates that can happen within a physical classroom. "You don't have the in-person camaraderie I guess." However, he explained that he still felt more connected in a blended environment than a purely online class:

I think the HoT session definitely felt a little more authentic and closer to an in-person session just by the fact that you're looking and seeing students in a classroom and you see the teacher there walking around the room. I definitely think that felt a little bit more personal than a truly online setting.

The need for physical connection was echoed by on-campus students after a design decision in the third iteration to separate the breakout groups into only online or on-campus students. For on-campus students, the removal of technology allowed them to communicate in a more natural way with one another. Annie, an on-campus student, noted, "I think we made better progress within the on-campus groups because we were able to more easily go round the table and openly discuss things and read each other's body language."

The instructor also concurred with this need for physical connections. She described an "energy" that is only present in face-to-face classroom settings where there is nonverbal feedback. However, she felt that this classroom energy can be replicated to some extent in blended synchronous environments, even for students who are not in the classroom. She explained that "there's a performance element to teaching that you... that you just lose in a purely online environment, although I think there's a stronger benefit for people that actually come onto campus for a HoT class, I feel like there is some benefit for the online students, to benefit from the energy."

Dichotomizing Students. Even in merging the online and on-campus students via a blended synchronous environment, students still saw the class as two distinct groups. The sessions were called HoT, which from the outset divided the group into "here" or "there" students. This division kept the students from feeling like a unified class and caused some confusion, distraction, and, at times, hostility.

On-campus students often felt divided from the online students. In the feedback from the first iteration, when there were technical issues, one anonymous on-campus student was quite hostile toward the online students, saying: "I feel like the communication would have been more effective if it was just between us as a small group IN THE CLASSROOM [student emphasis]. Too much distraction and time wasted with people from the outside." Even during later iterations, when more students were using video and many technical issues were resolved, Mike explained that the online students felt like guests:

This is our class because you know you see the same people, you sit in the same seats, we're in pretty much the same groups paired to our learning capabilities... so you sort of have collaboration with these people and then the people who were online were just little black [outlines of] faces on WebEx.

Online students also felt a disconnect, particularly at times when there were technical issues with WebEx, and it was unknown to them what was going on in the classroom. George explained: "Being remote, you can't really see all the time what's happening. You kind of wonder what's going on." He further noted that by the nature of being physically present, the on-campus students seemed to be the priority: 
It may have felt like there was more discussion and focus on the in-person students than the remote or online students. Just because they're there and they can talk to them quickly and we're just kind of a name on the screen.

Whereas the on-campus student felt that the online students were outsiders or a distraction, online students felt, at times, as though they were excluded because of their virtual presence.

In the final iteration, when small groups were separated into either all on-campus or all online students, the on-campus students felt relief in being able to interact more naturally and without the possibility of technical issues. However, it further segregated the class, which was noted by Mike as problematic: "I did like the fact that the in-class people were grouped together, and the online people were grouped together. I liked that, but I miss the collaboration with the online people."

\section{Discussion}

The research team brainstormed technological design recommendations for each assertion that would improve the learner experience to better support co-presence. Each of the sections that follow describe these design recommendations in relation to this study's assertions and to findings from other researchers investigating blended synchronous learning.

\section{Streamline Technology to Create a More Seamless Learning Experience}

Creating blended synchronous learning environments inherently requires the use of technology. However, based on the experiences of the instructor and students in this study, the design recommendation for creating a more seamless experience is to intentionally select and integrate only the technology deemed necessary to support pedagogy and create co-presence between and among learners. This design recommendation relates to three assertions: (a) technology as a limitation, (b) awkwardness in merging environments, and (c) the vulnerability of transitions. Some technology issues, awkwardness, and feelings of being overwhelmed by the technology can be avoided by eliminating unnecessary technology (e.g., number of microphones) and reducing the number of transitions between whole-class discussions and small groups where technology issues were more likely to occur. Decisions to streamline technology can lead to marked improvements in the learner experience. For example, in this study, although it was necessary to use a web-conferencing tool to connect on-campus and online students, it was not a necessity for every student attending on campus to log into the tool as they did during the first and second iteration. By only having the online students log into the web conferencing tool, the interactions between students in the classroom became more seamless and there were less technological disruptions. Web conferencing tools that enable smooth transitions to breakout groups that allow for use of video are recommended for blended synchronous environments.

Challenges with technology are a common finding in studies involving blended synchronous environments (Bell et al., 2014; Bower et. al., 2015; Butz \& Stupnisky, 2016; Park \& Bonk, 2007). Streamlining technology addresses these issues and could reduce the time for training students in the use of the software as recommended by Bower et. al. (2015), which may not always be practical or feasible for the instructor. At this point, some degree of technological disruption is likely unavoidable given the relative novelty of blended synchronous sessions and the technology currently available. The potential of blended synchronous environments will be dependent on the continued evolution of technology that affords instructors the ability to let 
pedagogy drive their decisions regarding how to use technology rather than making decisions based solely on a desire to avoid disruptions. This may also require some instructors to expand their knowledge of digital pedagogies and experiment with new approaches (Hastie, Hung, Chen \& Kinshuk, 2010) or redesign activities to work in this setting (Wang \& Huang, 2018; Wang et al., 2017; 2018).

\section{Enhance Co-Presence Through Visual or Physical Connections and Inclusive Language}

The second design recommendation, to enhance co-presence through visual or physical connections and inclusive language, emerged from two assertions: (a) seeing is believing and (b) dichotomizing students. Without being able to see one another, students did not feel like part of a cohesive group. Optimally, the faces of all online students should be displayed throughout the session. The use of video should be as seamless as possible and cameras that require students to pass a pendant should be avoided. Separating breakout groups into all on-campus and all online students, which seems to counter the notion of creating co-presence, allows on-campus students to communicate directly with one another rather than through a mediated technology in addition to allowing online students to have a common shared experience, decreasing frustration for all. Eliminating the language of here or there from the description of the course and providing more whole-group experiences with all on-campus and online students together can also further increase co-presence.

In a similar study, in which online students were brought into the physical classroom via Skype, Cunningham (2014) makes a case that richer media that enables cues like facial presence, gaze, and posture, allow for increased social presence. Other studies have recommended using 3D virtual reality in order to make the experience more authentic for online students (Bower et al., 2017; Wang \& Huang, 2018; Wang et al., 2018). The physical/visual still matters (Barad, 2003) because it is preferred by students, so increasing both physical/visual connections as well as limiting language that divides the students can support co-presence in blended synchronous environments.

\section{Limitations}

Additional research is necessary before any definitive claims about the technological design of blended synchronous learning environments can be made. Case studies are highly contextualized and feature a small sample size. Although this method results in rich descriptions of a specific experience, the generalizability of those results is limited. The findings of this study aligned and expanded on existing research, however, and additional studies are needed to explore the suitability of WebEx in blended synchronous environments. In addition, the exploration of other web conferencing tools that may better support seamless, active learning that meets the needs of both online and on-campus students is necessary. Perhaps commercial web conferencing tools typically created for business settings will not work to create the co-presence desired by students in higher education settings. Therefore, the design of new tools created specifically for educational settings should be another focus of future research. Lastly, in addition to web-conferencing tools, how to leverage the additional technology used in the classroom needs to be systematically studied. This should include an evaluation of how different uses of camera and audio equipment impacts the learner experience and co-presence. 


\section{Conclusion}

This study made several contributions to the exploration for the optimal technological design of blended synchronous environments. The findings helped to articulate the requirements for a web conferencing tool needed to support this type of learning. Technology limitations continue to be an ever-present issue in blended synchronous learning (e.g., Bell et al., 2014; Bower et al., 2015; Park \& Bonk, 2007; Wang \& Huang, 2018; Wang et al., 2018), however, this study found success in streamlining the use of technology and reducing the number of transitions. Findings also indicated that enabling or simulating physical connections through rich media could help create a feeling of co-presence among participants, which concurred with earlier research studies (Szeto \& Cheng, 2016).

Blended synchronous learning has the potential to increase students' co-presence in support of a seamless learner experience and improve upon the flexibility and accessibility of course offerings if designed well. This case study provided recommendations for how best to design the technology of a blended synchronous learning environment and identified areas of future research that will help realize that potential. 


\section{References}

Bell, J., Sawaya, S., \& Cain, W. (2014). Synchromodal classes: Designing for shared learning experiences between face-to-face and online students. International Journal of Designs for Learning, 5, 68-82. doi: 10.14434/ijdl.v5i1.12657

Bower, M., Dalgarno, B., Kennedy, G. E., Lee, M. J., \& Kenney, J. (2015). Design and implementation factors in blended synchronous learning environments: Outcomes from a cross-case analysis. Computers \& Education, 86, 1-17. doi: 10.1016/j.compedu.2015.03.0060360-1315

Bower, M., Lee, M. J., \& Dalgarno, B. (2017). Collaborative learning across physical and virtual worlds: Factors supporting and constraining learners in a blended reality environment. British Journal of Educational Technology, 48(2), 407-430. doi: $10.1111 /$ bjet.12435

Bulu, S. T. (2012). Place presence, social presence, co-presence, and satisfaction in virtual worlds. Computers \& Education, 58(1), 154-161.doi: 10.1016/j.compedu.2011.08.024

Butz, N. T., \& Stupnisky, R. H. (2016). A mixed methods study of graduate students' selfdetermined motivation in synchronous hybrid learning environments. The Internet and Higher Education, 28, 85-95. doi: 10.1016/j.iheduc.2015.10.003

Charmaz, K. (2006). Constructing grounded theory: A practical guide through qualitative analysis. Sage Publications. doi: 10.3402/qhw.v1i3.4932

Cunningham, U. (2014). Teaching the disembodied: Othering and activity systems in a blended synchronous learning situation. The International Review of Research in Open and Distributed Learning, 15(6), 33-51. doi: 10.19173/irrodl.v15i6.1793

Dempsey, N. P. (2010). Stimulated recall interviews in ethnography. Qualitative Sociology, 33(3), 349-367. doi: 10.1007/s11133-010-9157-x

Erickson, F. (1986). Qualitative methods in research on teaching. In M. Wittrock (Ed.), Handbook of research on teaching (3rd ed., pp. 119-161). MacMillan.

Hastie, M., Hung, I. C., Chen, N. S., \& Kinshuk. (2010). A blended synchronous learning model for educational international collaboration. Innovations in Education and Teaching International, 47(1), 9-24. doi: 10.1080/14703290903525812

McDonald, J.P., Zydney, J.M., Dichter, A., \& McDonald, B. (2012). Going online with protocols: New tools for teaching and learning. NY: Teachers College Press.

Merriam, S. B. (2001). Qualitative research and case study applications in education. JosseyBass Publishers.

Moore, M. G. (2013). Handbook of distance education. Taylor and Francis.

Para, J. L., \& Abdelmalak, M. (2016). Expanding learning opportunities for graduate students with HyFlex course design. International Journal of Online Pedagogy and Course Design, 6(4), 19-37. doi: 10.4018/IJOPCD.2016100102

Park, Y. J., \& Bonk, C. J. (2007). Synchronous learning experiences: Distance and residential learners' perspectives in a blended graduate course. Journal of Interactive Online Learning, 6(3), 245-264. http://www.ncolr.org/issues/jiol/v6/n3 
Romero-Hall, E., \& Vicentini, C. (2017). Examining distance learners in hybrid synchronous instruction. Successes and challenges. Online Learning, 21(4), 141-157. doi:

10.24059/olj.v21i4:1258

Szeto, E. (2015). Community of Inquiry as an instructional approach: What effects of teaching, social and cognitive presences are there in blended synchronous learning and teaching? Computers \& Education, 81, 191-201. doi: 10.1016/j.compedu.2014.10.015

Szeto, E., \& Cheng, A. Y. (2016). Towards a framework of interactions in a blended synchronous learning environment: what effects are there on students' social presence experience? Interactive Learning Environments, 24(3), 487-503. doi: $10.1080 / 10494820.2014 .881391$

Tolman, D. L., \& Brydon-Miller, M. (2001). From subject to subjectivities: A handbook of interpretive and participatory methods. New York University Press.

Wang, Q., \& Huang, C. (2018). Pedagogical, social and technical designs of a blended synchronous learning environment. British Journal of Educational Technology, 49(3), 451462. doi:10.1111/bjet. 12558

Wang, Q., Huang, C., \& Quek, C. L. (2018). Students' perspectives on the design and implementation of a blended synchronous learning environment. Australasian Journal of Educational Technology, 34(1), 1-13. doi:10.14742/ajet.3404

Wong, L.-H., \& Looi, C.-K. (2011). What seams do we remove in mobile assisted seamless learning? A critical review of the literature. Computers \& Education, 57(4), 2364-2381.

Wang, Q., Quek, C. L., \& Hu, X. (2017). Designing and improving a blended synchronous learning environment: An educational design research. The International Review of Research in Open and Distributed Learning, 18(3), 99-118. doi: 10.19173/irrodl.v18i3.3034

White, C. P., Ramirez, R., Smith, J. G., \& Plonowski, L. (2010). Simultaneous delivery of a faceto-face course to on-campus and remote off-campus students. TechTrends, 54(4), 34-40. doi:10.1007/s11528-010-0418-z

Yin, R. K. (2003). Case study research: Design and methods (3rd ed.). Sage.

Zydney, J.M., McKinney, P., Lindberg, R., \& Schmidt, M. (2019). Here or there instruction: Lessons learned in implementing innovative approaches to blended synchronous learning. TechTrends. 62(2),123-132. doi: 10.1007/s11528-018-0344-Z

Zydney, J. M. \& Warner, Z. \& Angelone, L. (2020). Learning through experience: Using design based research to redesign protocols for blended synchronous learning environments. Computers \& Education, 143(103678). doi:10.1016/j.compedu.2019.103678. 


\section{Appendix A}

\section{Classroom Observation Form}

Instructor Name: Class Name:

Observer Name: Date/ Time:

\section{Classroom / Environment Description:}

Describe the classroom layout (e.g. rows of seats, chairs in U shape, table groupings, etc.), the people present, and ambient qualities (e.g., noises, amount of light, any noticeable smells, etc.) 
Directions: Record a log of events, activities, conversations that happened along with your own commentary on your thoughts and feelings.

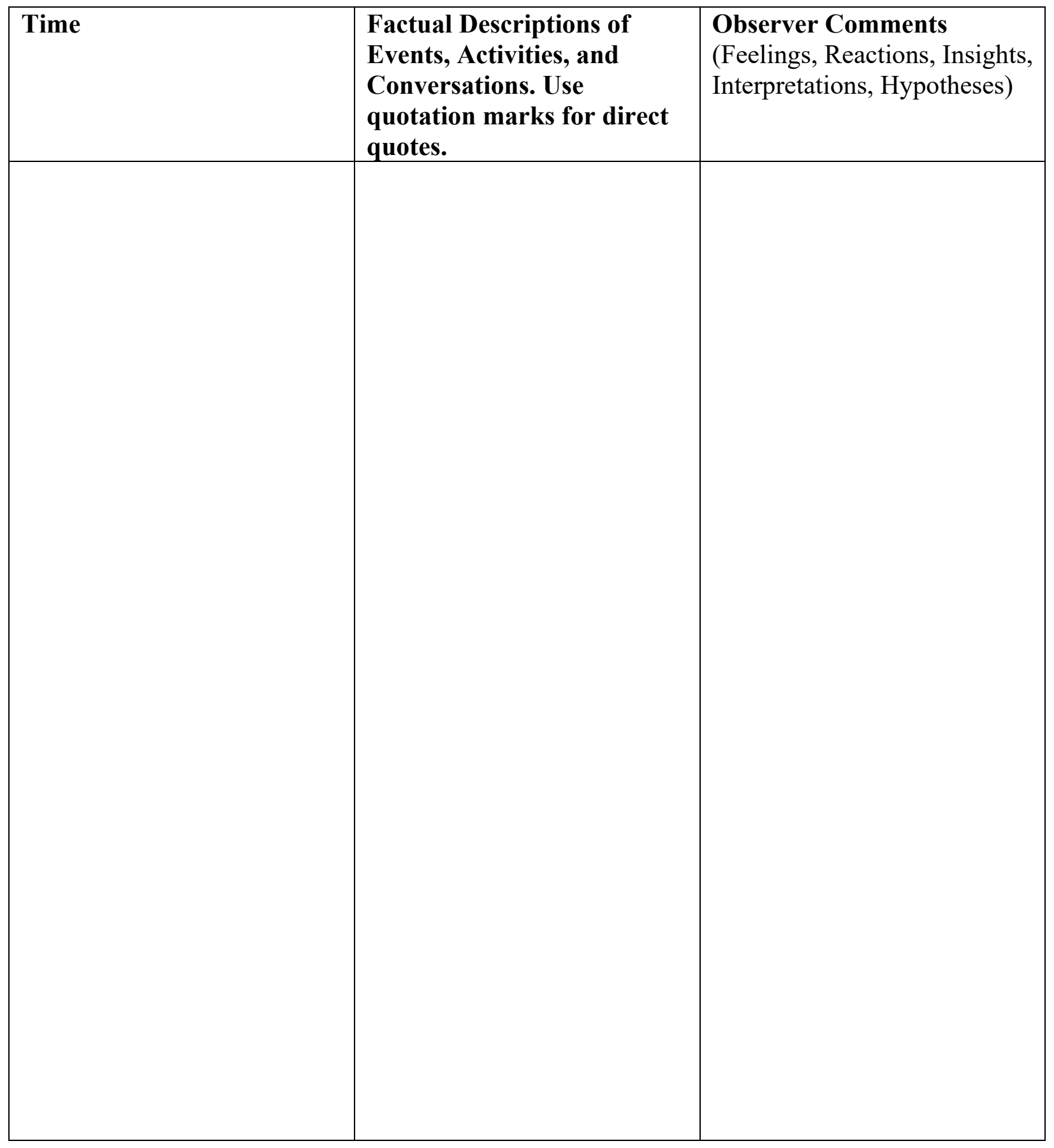

attach additional pages as needed. 


\section{Appendix B}

\section{Sample Interview Question}

At this point in the semester we have had 3 HoT sessions, and I would like to know more about your experiences in each of them. Since these sessions have spanned several months, I will briefly review what took place during each session and then ask you to reflect on your experience. I may also ask you some follow-up questions along the way if there is something I think would be helpful for us to know more about, or if I want to make sure I am understanding you correctly.

1. Session 1: For the first HoT session, everyone (both on and off-campus) logged in to the WebEx training session. The instructor began by introducing the course and what protocols are used for. For the main discussion, there was central mic set up on a rolling chair which moved around to pick up everyone's voices in the classroom. For the breakout sessions, everyone used headsets to participate in the discussion and turned their microphone on when they wanted to speak. For the discussions, the instructor controlled the breakout sessions by creating groups within the training session, which she restarted once because of technical issues. The protocol that we used for the discussion was called Marvin's Model, where the group answers several questions in Go Rounds (a specific technique of a protocol where each person talks one at a time in a specific order). The Go Rounds were about using Voicethread for our asynchronous discussions, what is was like to post in a medium that was comfortable/uncomfortable, and how you can apply this experience to your teaching. The instructor controlled the PowerPoint with the questions throughout the discussion so all of the groups had to progress at relatively the same pace. The facilitators were all physically in the classroom and asked each question to the group that the instructor posed. The instructor walked around to the group facilitators to check on progress. Time warnings were sent through the WebEx message system. After the discussion was completed, one person in each group (two were online students) led share outs of what you all created to represent your discussions.

What do you recall about your experience while the session was actually taking place?

Note: HoT stands for Here or There and was the acronym used in the course for blended synchronous sessions. 